\title{
Macrophage Functions Are Regulated by Murine Decidual and Tumor Extracellular Matrices
}

Dianne B. McKay, " Miguel A. Vazquez, ${ }^{5}$ Raymond W. Redline, ${ }^{\ddagger}$ and Christopher Y. Lu

With the technical assistance of Sherri R. Schmalzried and Colleen $\mathbf{M}$. Shea

Departments of *Medicine (Renal Division) and ${ }^{\ddagger}$ Pathology (Women’' Division), Brigham and Women’s Hospital,

Boston, Massachusetts; Division of ${ }^{\S}$ Nephrology, Department of Internal Medicine,

University of Texas Southwestern Medical School, Dallas, Texas 75235-8856

\begin{abstract}
Because of their paternal antigens, the fetus and placenta may be considered an allograft in the maternal host. Understanding the mechanisms which prevent maternal immunological rejection of the fetus remains a fundamental unsolved problem in immunology. We have previously reported that macrophages are inhibited by maternal decidual stromal cells residing at the maternal-fetal interface. In view of the central role of macrophages in cell-mediated immunity, this inhibition may contribute to preventing maternal antifetal responses. We now report that it was the solid phase signals embedded in the extracellular matrix (ECM) made by decidual cells which are responsible for inhibiting macrophage-mediated lysis of TNF-alpha-resistant P815 mastocytoma cells. The latter macrophage function is acquired after stimulation by interferon gamma and endotoxin. All these macrophage functions were also inhibited by ECM isolated from the Engelberth-Holm-Swarme (EHS) tumor. This tumor ECM has a similar biochemical composition to decidual ECM. This ECM inhibited the effector, as opposed to the stimulator, phase of macrophage-mediated tumor lysis. Laminin, type IV collagen, and heparan sulfate proteoglycans, the major known components of decidual and EHS ECMs, did not inhibit the above macrophage functions. Altogether these data indicate that macrophages were inhibited by solid phase signals embedded in decidual and EHS ECMs. Whether the solid phase signals in these two ECMs are biochemically identical remains to be determined. To our knowledge, such signals are a novel pathway of inhibiting macrophage functions which may be important in understanding the maternal-fetal immunologic relationship, and the pathogenesis of perinatal infections. Furthermore, the ability of EHS tumor ECM to inhibit macrophage functions may indicate that some tumors may defend themselves against host macrophage responses using solid phase signals. This may be important in understanding some host-tumor relationships. (J. Clin. Invest. 1992. 89:134-142.) Key words: listeria monocytogenes $\bullet$ interferon gamma $\bullet$ endotoxin • placenta
\end{abstract}

Address correspondence to Christopher Y. Lu, M.D., Division of Nephrology, Dept. of Internal Medicine, UT Southwestern Medical Center, 5323 Harry Hines Blvd., Dallas, TX 75235-8856. 1991.

Received for publication 09 August 1990 and in revised form 01 July

J. Clin. Invest.

(C) The American Society for Clinical Investigation, Inc.

0021-9738/92/01/0134/09 \$2.00

Volume 89, January 1992, 134-142

\section{Introduction}

The decidua basalis is an important tissue from an immunologist's viewpoint. It is the tissue where the placenta is anchored to the transformed endometrial stroma of the maternal uterus and is the only uteroplacental region in mice where fetal class I MHC-expressing trophoblast cells are in intimate contact with maternal decidual cells (1). It is one of the few tissues in nature where semiallogeneic cells coexist without immunologic rejection, perhaps because maternal antifetal immune responses are inhibited (for reviews see 2-4).

Macrophages play an important role in allograft rejection (5), and we have recently demonstrated that their function in the decidua basalis is inhibited using a model of intrauterine infection by Listeria monocytogenes. This intracellular pathogen is an ideal probe for the local regulation of macrophage responses in the decidua basalis in vivo because Listeriosis is a powerful stimulus of macrophage activation. Furthermore, macrophages must actually enter infected tissue to destroy Listeria (6), and thus come under the influence of local regulatory factors in the tissue. We found that macrophage functions were inhibited in the decidua basalis $(7,8)$ and that this resulted in unrestrained bacterial proliferation at these sites. Inhibition of macrophage functions was restricted to the decidua basalis, and not a general property of pregnancy, because there was a vigorous effective antilisterial response in the maternal spleen and other organs.

The decidua basalis consists of maternal decidual cells and fetal trophoblast (1). Deciduoma of pseudopregnancy were used to demonstrate that maternal decidual cells alone could account for the inhibition of macrophage antilisterial responses in vivo (9). Such deciduoma closely mimic the decidua basalis of true pregnancy except that no fetally derived cells are present. Furthermore, decidual stromal cells form a "substratum" in vitro which consists of the cells themselves and their associated extracellular matrix (ECM). ${ }^{1}$ Solid phase inhibitors embedded in this substratum inhibited macrophage activation (10). The effect of such solid phase, rather than freely diffusible, signals can be spatially limited. This would have significant advantages in an anatomically complex area such as the maternal-fetal interface. Indeed, macrophages are inhibited only in the decidua basalis where maternal and fetal cells are intimately intermixed. In contrast, other immediately adjacent regions, which contain exclusively maternal cells, retain the capacity to support macrophage responses (8).

To our knowledge, this was the first demonstration that solid phase inhibitors regulate macrophage functions. How-

1. Abbreviations used in this paper: ECM, extracellular matrix; EHS, Engelberth-Holm-Swarm tumor. 
ever, it was not known if the solid phase inhibitor was on the decidual cell surface or in the ECM. Furthermore, a labile soluble inhibitor secreted by decidual stromal cells and active only a short distance from these cells could not formally be excluded.

We now show that it is the decidual extracellular matrix (ECM) which inhibits macrophage functions. We also investigated the ECM produced by the Englebreth-Holm-Swarme (EHS) tumor because it is biochemically similar to decidual ECM (11-13). We found that EHS ECM also inhibited macrophage activation in vitro as well as antilisterial macrophage responses in vivo.

\section{Methods}

Animals. C57Bl/6J mice (Listeria-resistant $[14,15])$ and female Sprague-Dawley rats of $\sim 6$ wk of age were purchased from Jackson Laboratories, Bar Harbor, ME, and Charles River Laboratories, Inc., Wilmington, MA, respectively. All animals were housed in the Harvard Medical School Cancer Research Facility, and later in the $Y$ and $J$ Facilities at the University of Texas Southwestern Medical School, and treated according to institutional and National Institutes of Health guidelines.

Listeria infection. Adult mice were immunized by one i.p. injection of $5 \times 10^{3}$ Listeria monocytogenes (EGD strain). Listeria were stored frozen at $-80^{\circ} \mathrm{C}$ in $20 \%$ glycerol in PBS, thawed, diluted in PBS, and quantified by plating on brain heart infusion agar (7). $14 \mathrm{~d}$ after immunization, the mice were placed under Avertin anesthesia, and a small incision was made over each hamstring muscle. Under direct visualization, Listeria were injected into the EHS tumor residing in the hamstring muscle of one hindlimb and into the contralateral, non-tumorbearing hamstring muscle of the same mouse. In this way, each animal served as its own control. 3-6 d later, the mice were killed by cervical dislocation and the EHS tumor, and contralateral hamstring muscle harvested under sterile conditions. Each organ was homogenized in cold sterile PBS, plated onto brain-heart-infusion-agar plates, and the number of Listeria colonies determined as described previously (7).

Engleberth-Holm-Swarm (EHS) tumor. The EHS tumor, originally a gift from H. K. Kleinman (16), was serially passed in the right hamstring muscles of $\mathrm{C} 57 \mathrm{Bl} / 6$ mice by intramuscular injection of washed tumor homogenate.

Isolation of EHS ECM. Mice were sacrificed by cervical dislocation and the EHS tumor harvested under sterile conditions as previously described (16). All subsequent extraction steps were performed on ice, under sterile conditions, and in the presence of $0.002 \mathrm{M} \mathrm{N}$-ethylmaleimide and $0.0004 \mathrm{M}$ ethylenediaminetetraacetic acid. All glassware was thoroughly cleaned and then baked at $200^{\circ} \mathrm{C}$, dry heat, to inactivate any contaminating endotoxin (17). The EHS tumor was mechanically disrupted by three homogenizations in $3.4 \mathrm{M} \mathrm{NaCl}$ and $0.05 \mathrm{M}$ Trisbuffer-Tris(hydroxymethyl)aminomethane, $\mathrm{pH}$ 7.4. The resulting slurry was stirred overnight at $4^{\circ} \mathrm{C}$ with an equal volume of $2 \mathrm{M}$ urea and $0.05 \mathrm{M}$ Tris, $\mathrm{pH} 7.4$. In the morning the slurry was centrifuged at $26,500 \mathrm{~g}$ for $20 \mathrm{~min}$, and the supernatant saved on ice. The residual pellet was redissolved in the sterile, cold $\mathrm{NaCl}$-Tris buffer solution and centrifuged again. The supernatants were combined and dialyzed ( $m o l$ wt cut-off $6,000-8,000 \mathrm{kD})$ against sterile, Tris-saline $(0.05 \mathrm{M}$ Tris and $0.15 \mathrm{M} \mathrm{NaCl}$ ) for $6 \mathrm{~h}$ at $4^{\circ} \mathrm{C}$, total of three exchanges. The solution was then dialyzed overnight, at $4^{\circ} \mathrm{C}$, against sterile DMEM (Gibco, Grand Island, NY). In the morning the contents of the dialysis bag were removed and the EHS ECM either used immediately or frozen at $-70^{\circ} \mathrm{C}$. EHS ECM gels formed when 50 lambda of the preparation were placed into microwells of a 96-well microtiter tray, and were warmed to $37^{\circ} \mathrm{C}$. Except for Fig. 2, the gels were washed with media before use.

Preparation of deciduoma and isolation of decidual ECM. Female Sprague-Dawley rats were rested in our animal facility for $\sim 1 \mathrm{wk}$, underwent bilateral oophorectomy under methohexital-sodium (Brevital) anesthesia, and were allowed to recover for $6 \mathrm{~d}$. Deciduoma were then induced as previously described (18). Rats received subcutaneous injections of $0.2 \mu \mathrm{g}$ estradiol (Sigma Chemical Co., St. Louis, MO) dissolved in sterile peanut oil on days $1-3$. On day 3 , they received an additional subcutaneous injection of $0.2 \mu \mathrm{g}$ estradiol and $1 \mathrm{mg}$ progesterone (Sigma Chemical Co.) at sunset. No injections were given on days 4 and 5. On days 6 and 7, morning injections of $0.1 \mu \mathrm{g}$ estradiol and $4 \mathrm{mg}$ progesterone were given. An additional $0.1 \mu \mathrm{g}$ of estradiol was given on the afternoon of day 7 . On day 8 , the rats received an injection of $0.1 \mu \mathrm{g}$ estradiol and $4 \mathrm{mg}$ progesterone in the morning, and then in the afternoon underwent a laparotomy under Brevital anesthesia. 100 lambda peanut oil was injected into each uterine horn, and 3-0 silk ligatures were then placed at the ends of each uterine horn to prevent the oil from escaping. On days 9-13, morning injections of $0.1 \mu \mathrm{g}$ estradiol and $4 \mathrm{mg}$ progesterone were given. On day 13 the animals were killed by $\mathrm{CO}_{2}$ narcosis or an overdose of Brevital. The decidualized uterine horns were removed under sterile conditions, placed in sterile media on ice and the deciduoma dissected away from the uterine walls. The decidual ECM was then isolated in the same manner as the EHS tumor. See above.

ECM components. Type I collagen gels were prepared by mixing one part sterile $0.2 \mathrm{M} \mathrm{NaOH}$ and one part 10× HBSS (Gibco) with eight parts Type I collagen (rat tail collagen, Vitrogen 100; Collagen Corp., Palo Alto, CA). The concentration of collagen was $2.4 \mathrm{mg} / \mathrm{ml}$, and was a solution at $4^{\circ} \mathrm{C}$. 50 lambda aliquots were added to each well of a 96-well microtiter plate, and allowed to gel by warming to $37^{\circ} \mathrm{C}$ for $2 \mathrm{~h}$. In some experiments, decidual or EHS ECM components were added to the Type I collagen solution at $4^{\circ} \mathrm{C}$, and the mixture allowed to gel upon warming.

Laminin and Type IV collagen (both purified from EHS tumor; Collaborative Research, Bedford, MA) were prepared from frozen stock, diluted to desired concentration, used to coat the bottom of tissue culture wells according to the manufacturer's instructions. In some experiments laminin and type IV collagen were individually plated on bacteriologic plastic plates. Adequacy of coating was confirmed by the change in macrophage morphology from their appearance on uncoated surfaces. For instance, on bacteriologic plates macrophages are typically rounded. When the surface is coated with laminin, the macrophages become spindle-shaped in the presence of activation signals. The same change in morphology was seen with type IV collagen coated plates.

Treatment of EHS ECM with specific glycosaminoglycan hydrolases. Gels formed from EHS ECM components were treated for $2 \mathrm{~h}$ with a mixture of Heparinase $(125 \mathrm{mU} / \mathrm{ml}$, Flavobacterium heparinum; Sigma Chemical Co.) and heparitinase (125 mU/ml, Flavobacterium heparinum; ICN Laboratories, Lisle, IL). Longer periods of treatment, i.e., overnight, resulted in disruption of the gel. After treatment with the enzymes, each well was carefully washed with warm culture medium (five times) to remove any residual enzyme and cleaved products. In other experiments, the EHS ECM gel was also treated with chondroitinase ABC $(250 \mathrm{mU} / \mathrm{ml}$, proteus vulgaris; Sigma Chemical Co.) and hyaluronidase $(250 \mathrm{mU} / \mathrm{ml}$, streptomyces hyalurolyticus and bovine testis; Sigma Chemical Co.).

Macrophages. RAW 264.7 macrophages (19), originally from the American Type Culture Collection, Rockville, MD, are an Abelson murine leukemia virus-transformed macrophage cell line which expresses many macrophage activities including lysozyme secretion, phagocytosis and killing of P815 tumor targets $(19,20)$. The RAW 264.7-macrophages are maintained in our laboratory in continuous culture at $37^{\circ} \mathrm{C}, 95 \% \mathrm{O}_{2}, 5 \% \mathrm{CO}_{2}$ in RPMI 1640 supplemented with $10 \%$ FCS, $5 \mathrm{mM} N$-2-hydroxyethylpiperazine- $N$ '-2-ethanesulfonic acid (Hepes), $2 \mathrm{mM}$ L-glutamine, $5 \mathrm{U} / 100 \mathrm{ml}$ penicillin $\mathrm{G}$, and $50 \mu \mathrm{g} / 100$ $\mathrm{ml}$ streptomycin sulfate. Peritoneal macrophages were harvested $4 \mathrm{~d}$ after an i.p. injection of thioglycollate.

Cytotoxicity assay. Details of the tumor killing assay have been reported elsewhere (10). EHS and decidual ECM, and Type I collagen are in solution at $4^{\circ} \mathrm{C}$, but form a gel when warmed to room temperature. 50 lambda of these solutions were placed in individual wells of a 96-well microtiter plate. Gels formed when the plates were warmed. 
$3.75 \times 10^{4}$ RAW 264.7 or peritoneal macrophages were placed upon these gels, directly upon the tissue culture plastic well bottoms, or upon plastic coated with Type IV collagen, or laminin, see above. No attempt was made to separate adherent from nonadherent cells. The same number of macrophages was therefore present on the various surfaces. The RAW 264.7 was essentially $100 \%$ macrophagelike cells and the thioglycollate-elicited peritoneal exudate was over $90 \%$ macrophages as determined by staining with the antimacrophage monoclonal antibody F4/80 (21). The macrophages were stimulated for $8 \mathrm{~h}$ with recombinant rat interferon gamma (IFN-gamma; Amgen, Thousand Oaks, CA) plus LPS (E. coli endotoxin, L3254; Sigma Chemical Co.). 2 $\times 10^{4}$ '1'In-labeled P815 mastocytoma cells were then added to each well. $18 \mathrm{~h}$ later ${ }^{111}$ In released from lysed P815 tumor cells was determined using a gamma counter. The P815 mastocytoma cells, maintained in continuous culture in our laboratory, were labeled by a 15-minute incubation with ${ }^{111}$ Indium oxine (Amersham Corp., Arlington Heights, IL).

The "cytotoxicity" was calculated from the formula cytotoxicity $=100 \times(X C-S R / T C-S R)$, where $X C=$ experimental counts, $S R$ $=$ spontaneous release, and $T C=$ total counts added to the well.

TGF beta 1. TGF beta 1 isolated from porcine platelets was obtained from $\mathrm{R}$ and D Systems, Minneapolis, MN. This preparation contained $50 \mu \mathrm{g} \mathrm{BSA} / 1 \mu \mathrm{g}$ TGF beta 1 . It was activated in small volume of $4 \mu \mathrm{M} \mathrm{HCl}$ plus $1 \mathrm{mg} / \mathrm{ml} \mathrm{BSA}$, and then diluted in RPMI 1640 .

\section{Results}

Decidual and EHS ECM inhibit macrophage-mediated lysis of P815 tumor targets in vitro. The ability of macrophages to lyse TNF-alpha-resistant (22) P815 mastocytoma cells is a readily measured effector function which is often correlated with the ability of macrophages to destroy the intracellular pathogen Listeria monocytogenes (23). Macrophages, including the RAW 264.7 macrophage line (20), lyse P815 targets after priming by lymphokines, such as IFN-gamma, and triggering by a second signal such as endotoxin (for review see 24). Lysis of ${ }^{111}$ In-labeled P815 is detected by measuring ${ }^{111}$ In released into the media by lysed P815 tumor cells. We previously demonstrated that the substratum formed by decidual stromal cells in vitro inhibited the effector phase of macrophage-mediated tumor lysis (10). This decidual substratum consisted of the cells and their associated ECM, and it was not known which contained the inhibitor. Fig. 1 shows that it was the decidual ECM which inhibited P815 lysis by RAW 264.7-macrophages. In this experiment, RAW 264.7-macrophages were placed upon gels formed by Type I collagen containing various amounts of decidual or EHS ECM components. Although Type I collagen is not a component of decidual or EHS ECM, it is a major component of nondecidualized endometrial and other interstitial tissues where macrophage antilisterial responses are normal $(8,11-13)$. Type I collagen is soluble at $4^{\circ} \mathrm{C}$ and forms a gel when warmed. RAW 264.7-macrophage do lyse P815 upon these gels, and this may reflect competent macrophage functions known to occur in vivo in interstitial tissues which contain Type I collagen as a major constituent (8). Gels composed of varying amounts of decidual or EHS ECM components may be formed by mixing these with Type I collagen at $4^{\circ} \mathrm{C}$. These mixtures gel when warmed. The ability of RAW 264.7-macrophages to lyse P815 was inhibited upon gels containing decidual ECM. Inhibition decreased in a dose-dependent fashion as the proportion of decidual ECM in the Type I collagen gels decreased. Control experiments established that dilution of Type I collagen gels with up to $80 \%$ media did not change their

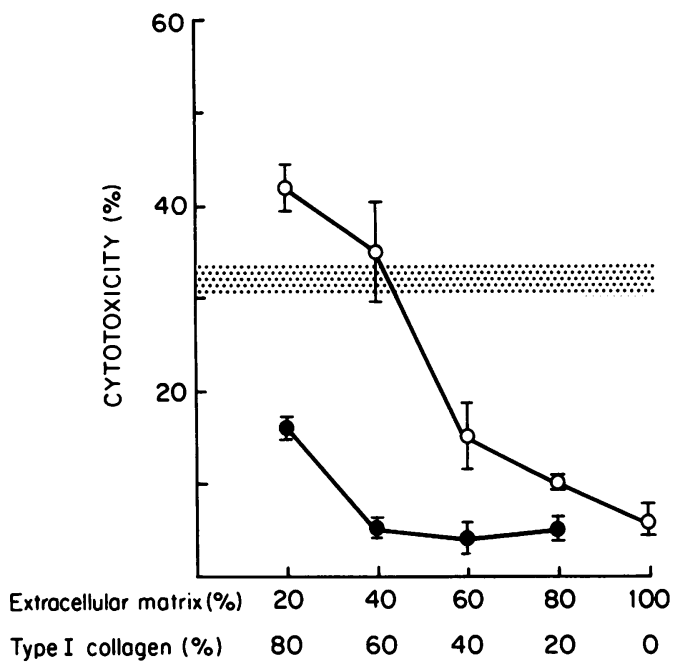

Figure 1. Decidual and EHS ECM both inhibit macrophage-mediated lysis of P815 tumor targets. Various amounts of decidual (solid circles) or EHS (open circles) ECM were incorporated into Type I collagen gels. The percentages along the $x$-axis are the percent of the total protein of the gel. Example 1: a 100\% Type I collagen gel has a protein concentration of $2.4 \mathrm{mg} / \mathrm{ml}$, all of which is Type I collagen. Example 2: when EHS ECM components were diluted in Type I collagen, a $20 \%$ extracellular matrix gel had $20 \%$ of the $2.4 \mathrm{mg} / \mathrm{ml}$ protein as EHS ECM, and the remaining $80 \%$ protein as Type I collagen. The RAW 264.7 macrophages residing on these gels were stimulated with 100 $\mathrm{U} / \mathrm{ml} \mathrm{IFN-gamma} \mathrm{and} 2 \mu \mathrm{g} / \mathrm{ml}$ endotoxin, and then allowed to lyse ${ }^{111}$ In-labeled P815 tumor targets. The spontaneous release was 23\%. See Methods for definition of "cytotoxicity." Each point is the mean $\pm \mathrm{SE}$ of three determinations. The shaded area is macrophagemediated cytolysis upon a $100 \%$ Type I collagen gel \pm SE. The results are representative of four different experiments.

ability to support RAW 264.7 macrophage-mediated tumor lysis.

Further investigation was limited by the small amounts of decidual ECM obtainable from rats. To illustrate this problem, we found that deciduoma from 12 rats yielded only $2.4 \mathrm{mg}$ of decidual ECM protein. Fortunately, ECMs formed by decidua and EHS tumor are similar biochemically in that they are both of the basement-membrane type and contain the following major components: laminin, type IV collagen, and heparan sulfate proteoglycans. Unlike the ECM of most interstitial spaces elsewhere in the body, decidual and EHS ECMs contain little type I collagen and fibronectin (11-13). Essentially unlimited amounts of EHS tumor can be grown in mice, and we found that gels containing EHS ECM also inhibited RAW 264.7 macrophage-mediated tumor lysis. Although in Fig. 1 the inhibition by EHS ECM was less pronounced than that by decidual ECM, it was potent and reproducible (see Figs. 2-4, 6-10).

EHS ECM components are soluble at $4^{\circ} \mathrm{C}$, physiologic ionic strength, and $\mathrm{pH}$, but form a gel when warmed. The formation of this gel is thought to represent reconstitution of the basement-membrane ECM for the following two reasons. The ultrastructure appears as thin interconnected sheets and resembles the lamina densa of basement membranes. The major components-laminin, type IV collagen, and heparan sulfate proteoglycan - polymerize in defined proportions (16). It is reasonable to assume that decidual ECM, having a similar biochemical composition (11-13), often forms gels for the same reasons. However, some preparations of decidual ECM 
were so dilute that no gel formed. If this occurred, the decidual ECM components were incorporated into Type I collagen gels and were found to be inhibitory.

The procedure used to extract ECM from EHS tumor and decidua was designed to isolate components insoluble at physiologic temperature, ionic strength, and pH (see Methods). However, we thought it important to exclude the possibility that a soluble inhibitor was diffusing out of the gels formed by these ECMs. Fig. 2 indicates that the inhibitor remained associated with the EHS ECM gel despite repeated washing $(A)$, and was not released into the media $(B)$. Altogether these data indicate that inhibition of RAW 264.7 macrophage-mediated tumor lysis upon EHS ECM was not due to previously described soluble macrophage "deactivating" factors secreted by other tumors (25-27).

The following control experiments were also performed (data not shown). (a) RAW 264.7-macrophages were not killed by decidual or EHS ECMs. After an overnight incubation on these surfaces, these cells acquired full tumoricidal activity when resuspended, placed on tissue culture plastic, and stimulated by interferon gamma and endotoxin. (b) Detection of tumor lysis in this system depends on release of ${ }^{111}$ In into the
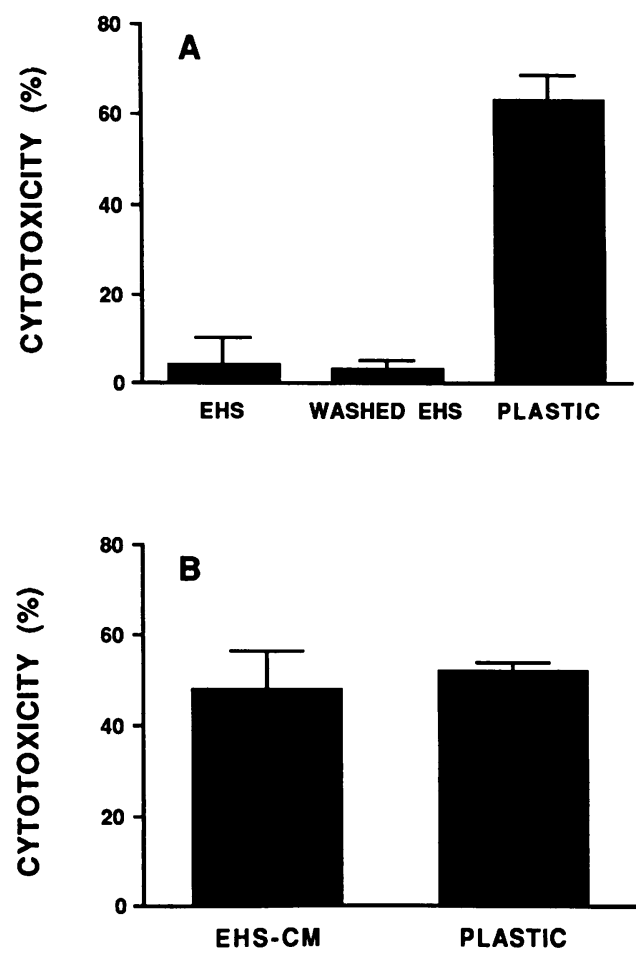

Figure 2. Inhibitor within gels formed by EHS ECM components is not soluble under physiologic conditions. $(A)$ Gel formed by EHS ECM was either not washed (EHS) or washed five times with media (WASHED EHS). RAW 264.7 macrophages were placed upon these gels or tissue culture plastic, stimulated by $1,000 \mathrm{U} / \mathrm{ml}$ IFN gamma and $0.2 \mu \mathrm{g} / \mathrm{ml}$ endotoxin, and then allowed to lyse ${ }^{111}$ In-labeled P815 targets. See Methods for definition of "cytotoxicity." $(B)$ Media was incubated upon EHS ECM gels overnight. This conditioned media $(E H S-C M)$ or fresh media (PLASTIC) was added to macrophages adhering to tissue culture plastic. After stimulation with the above doses of IFN gamma and endotoxin, these macrophages were allowed to lyse "11'In-labeled P815. Each point is the mean and standard error of triplicate determinations. culture medium where it can be sampled. We found that these ECMs did not sequester the radiolabel. Furthermore, the inability of RAW 264.7-macrophages to kill P815 on these surfaces was confirmed by Trypan Blue exclusion. (c) The decidual and EHS ECM gels did not bind or inactivate interferon gamma or endotoxin. Solutions of interferon gamma and endotoxin were fully active after incubation upon decidual and EHS ECM gels.

Effector phase of macrophage-mediated lysis of P815 inhibited by EHS ECM. Macrophage-mediated lysis of P815 may be divided into two phases: a "stimulator phase" when macrophages are primed by IFNg and triggered by endotoxin, and an "effector phase" when the P815 are lysed. The special properties of bacteriologic plastic were used to determine if EHS ECM inhibited the stimulatory or effector phase of macrophage-mediated tumor lysis. Unlike tissue culture plastic, bacteriologic plastic is a surface to which macrophages are only moderately adherent, from which they can be resuspended by vigorous washing, and upon which they can be activated to lyse P815 tumor targets. In Fig. 3, RAW 264.7-macrophages were stimulated with IFNg and endotoxin for $8 \mathrm{~h}$ upon either EHS ECM or bacteriologic plastic, resuspended, and washed. The macrophages were then placed together with P815 on either bacteriologic plastic or EHS ECM without further stimulation. RAW 264.7-macrophages stimulated on EHS ECM or bacteriologic plastic lysed P815 when subsequently placed on tissue culture plastic. On the otherhand, RAW 264.7-macrophages stimulated on EHS ECM or bacteriologic plastic did not lyse P815 when subsequently placed on EHS ECM. This data indicates that EHS ECM inhibits the effector phase of macrophage-mediated tumor lysis. Control RAW 264.7 macrophages, which were stimulated for a suboptimal time of $30 \mathrm{~min}$ on EHS ECM, washed, and then placed on tissue culture plastic, did not lyse P815. This excluded physical carryover of trace amounts of IFNg and endotoxin, remaining after the wash step, as a cause for lytic activity after transfer. We cannot yet formally exclude the possibility that instead of inhibiting macrophages, EHS ECM acts directly upon P815 making them resistant to lysis.

EHS ECM also inhibits peritoneal macrophage-mediated tumor lysis. Although RAW 264.7-macrophages are similar to peritoneal macrophages in most aspects, phagocytic ability, lysozyme secretion, and activation by interferon gamma and endotoxin $(19,20,28)$, there are some differences $(29)$. We therefore compared the effects of tissue culture plastic, EHS ECM, and Type I collagen gels on tumor lysis by RAW 264.7 and peritoneal macrophages. Fig. 4 shows that the behavior of these two populations of macrophages is similar. Tumor lysis occurred on tissue culture plastic and Type I collagen gels, but not on EHS ECM.

Defective antilisterial response localized to the EHS tumor in vivo. The above data indicate that decidual (Fig. 1) and EHS (Figs. 1-4) ECM inhibited macrophage-mediated tumor lysis. We have previously shown that decidual tissue inhibits macrophage antilisterial responses in vivo (7-9). In Fig. 5, we asked if the inhibition of macrophage-mediated tumor lysis by EHS ECM in vitro was also correlated with inhibition of antilisterial responses in vivo. Equal numbers of Listeria were injected into EHS tumor residing in one hamstring muscle, and into the contralateral tumor-free hamstring muscle. Each mouse thus served as its own control. Listeria growth in the EHS tumor was, on the average, six to seven orders of magnitude greater than in tumor-free muscle. 


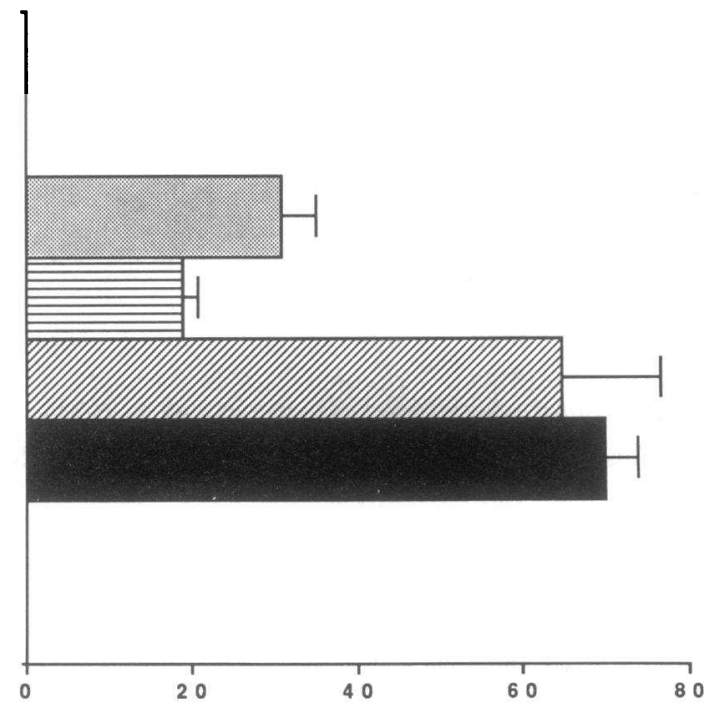

CYTOTOXICITY (\%)
Figure 3. Effector phase of macrophage-mediated tumor lysis is inhibited by EHS ECM. RAW 264.7 macrophages were stimulated with $1,000 \mathrm{U} / \mathrm{ml} \mathrm{rIFNg}$ and $20 \mu \mathrm{g} / \mathrm{ml}$ endotoxin while residing upon EHS ECM $(E H S)$ or bacteriologic plastic (PLASTIC). After $8 \mathrm{~h}$, the macrophages were resuspended, washed, and placed upon either PLASTIC or EHS. ${ }^{111}$ Inlabeled P815 were added and macrophage-mediated lysis of P815 measured. Each value is the mean and standard error of three measurements.
Effect of individual decidual and EHS ECM components on macrophage-mediated lysis of P815. Both decidual and EHS ECM preparations contain large amounts of laminin, type IV collagen, and heparan sulfate proteoglycans of the basement membrane type (11-13). We investigated the ability of laminin and Type IV collagen isolated from EHS tumor to inhibit RAW 264.7-macrophage-mediated tumor lysis. Figs. 6 and 7 indicate that plastic surfaces coated with these molecules did not inhibit RAW 264.7-macrophage-mediated lysis of P815.

To investigate the possibility that heparan sulfate glycosaminoglycans inhibited RAW 264.7-macrophage-mediated P815 lysis, the EHS ECM was incubated $2 \mathrm{~h}$ with a mixture of heparinase $(125 \mathrm{mU} / \mathrm{ml})$ and heparitinase $(125 \mathrm{mU} / \mathrm{ml})$, and washed. Although these hydrolases cleave heparan sulfate glycosaminoglycans from the core protein, we found that they did not prevent EHS ECM from inhibiting RAW 264.7-macrophage-mediated lysis of P815 (Fig. 8). We also found that chondroitinase and hyaluronidase also did not prevent EHS ECM from inhibiting macrophage-mediated tumor lysis.
We find that protease preparations do reverse the inhibitory activity of EHS ECM. These protease preparations are from bacteria and are heavily contaminated by endotoxin. This endotoxin potentiates macrophage-mediated tumor lysis (see Fig. 4) and is responsible for these results. Thus, boiling the protease preparation destroys the enzyme activity, but not the endotoxin. Boiling did not abolish the ability of the endotoxin-contaminated protease preparations to reverse the inhibitory activity of EHS ECM.

Recent data indicate that TGF beta is found in the gravid uterus and may play a role in inhibiting maternal antifetal responses $(30,31)$. This molecule may be bound in our EHS and decidual ECM preparations. However, Fig. 9 indicates that TGF beta-1 did not inhibit RAW 264.7-macrophage-mediated lysis of P815. Control experiments indicated that this TGF beta-1 preparation did have the expected inhibitory effect on lymphocyte proliferation (32), data not shown.

We have used a range of endotoxin concentrations in Figs. 1-4 and 6-9. The optimal dose varied depending on the partic-

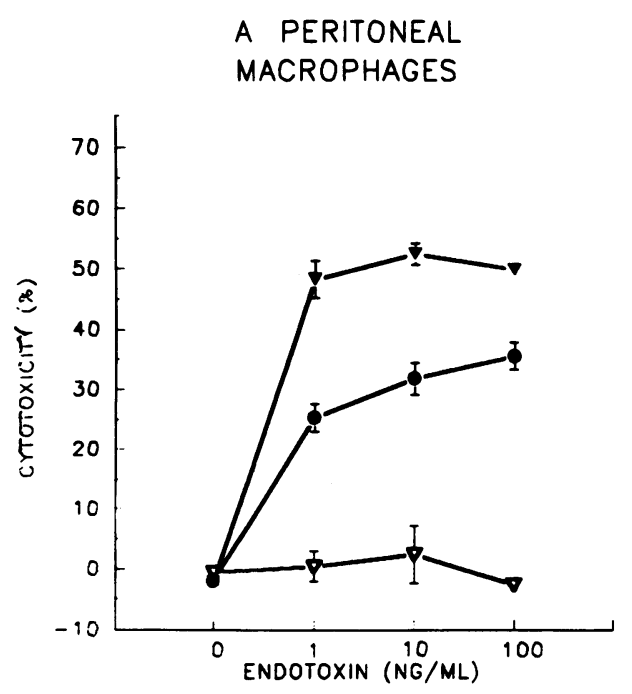

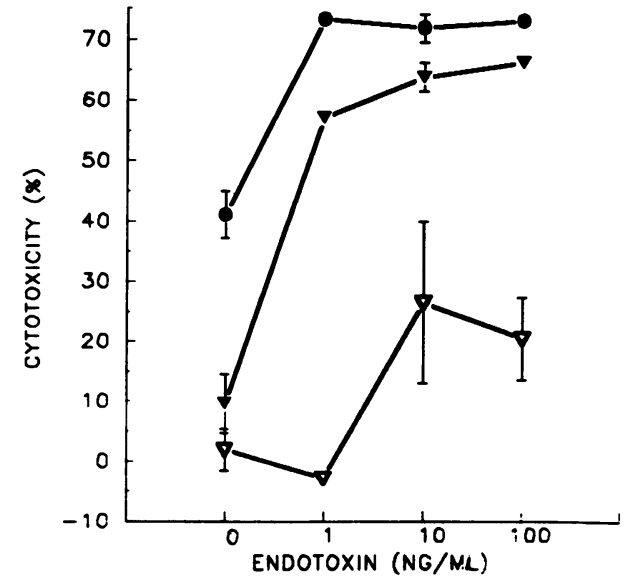

Figure 4. Tumor lysis by both RAW 264.7- and peritoneal macrophages is inhibited by EHS ECM. Macrophages cultured upon gels formed by Type I collagen (solid circles) or EHS ECM (open inverted triangles), or upon tissue culture plastic (solid inverted triangles). Macrophages were stimulated by $10 \mathrm{U} / \mathrm{ml}$ IFN-gamma and the endotoxin concentrations indicated on the abscissa, and then allowed to lyse ${ }^{111}$ In-labeled P815. (A) Peritoneal macrophages. (B) RAW 264.7 macrophages. 


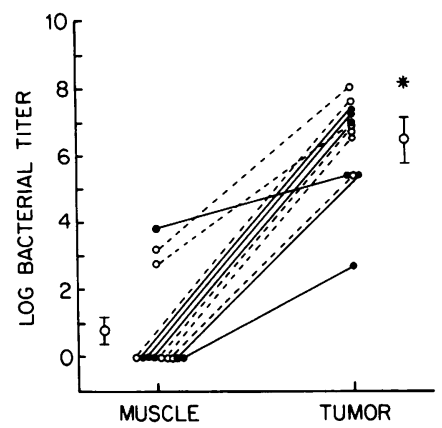

(open circles, dashed line) in other mice. mice. Each pair of symbols connected by a line represents results from a single mouse. When the innoculum was $5 \times 10^{3}$ bacteria, $0.97 \pm 0.6 \log _{10}$ Listeria were found in the tumor-free muscle after $6 \mathrm{~d}$, whereas $6.9 \pm 0.69 \log _{10}$ Listeria were in the EHS tumor. When the innoculums were increased to 5 $\times 10^{4}$ bacteria, there were $0.63 \pm 0.64$ and $6.1 \pm 0.69 \log _{10}$ Listeria in tumor-free muscle and EHS tumor, respectively. The results using these two innoculums of Listeria were not significantly different, and a combined mean $\pm \mathrm{SE}$ of Listeria growth in muscle versus EHS tumor was calculated. $P<0.001$ muscle versus EHS tumor.

ular preparation of endotoxin and macrophage. Although a similar range from $1 \mathrm{ng} / \mathrm{ml}$ (for example reference 33 ) to $10,000 \mathrm{ng} / \mathrm{ml}$ (for example, references 28,34 ) has been used in the literature, our macrophages tended to require higher endotoxin doses for an optimal response. This may be related to the much smaller numbers of macrophages used per well: 3.75 $\times 10^{4}$ /well in our experiments compared with $7.5-50 \times 10^{4} /$ well in published reports (for example, reference 29 ).

\section{Discussion}

The data from this report and previous publications from this laboratory (10) and others (35) are consistent with the hypothesis that macrophages are regulated by solid phase molecules embedded in the ECM. Furthermore, our data indicate that there are two broad classes of ECM: "inhibitory ECMs" which inhibit macrophage activation, and "permissive ECMs" which support macrophage activation. Three lines of evidence are consistent with this formulation.

(a) Evidence using two in vivo models. We have previously shown that there is a striking difference in the macrophage anti-listeria response in two immediately adjacent tissues of the gravid uterus. The decidua basalis where macrophage responses are profoundly inhibited, and the nondecidualized endometrium where the responses are vigorous (8). Solid phase, as opposed to soluble, diffusable molecules, would allow such spatial specificity in the location where macrophages are inhibited. The differences in macrophage function in the decidua basalis and nondecidualized endometrium may reflect the remarkably different ECMs of these two tissues $(11,12)$. The former is of the basement-membrane type and has, as major components, laminin, type IV collagen, and basement-membrane heparan sulfate proteoglycans. There is little fibronectin and type I collagen. The latter is of an interstitial type consisting predominantly of type I collagen and fibronectin. There is no laminin, type IV collagen, and basement-membrane heparan sulfate proteoglycans. According to our hypothesis, the decidual ECM is inhibitory for macrophage function, whereas the interstitial ECM is permissive. As a second in vivo model, we chose the EHS tumor because this tumor produces an ECM which is similar to decidual ECM. As predicted by our hypothesis, Fig. 5 demonstrates that the antilisterial responses were also inhibited in this tumor, but not in the interstitial spaces of the hindlimb without tumor. The advantage of these in vivo models is that they indicate a biologically important event: inhibition of antilisterial responses. The disadvantage is that in vivo models are complex, and any of a number of different mechanisms could account for the observed results. The two in vitro models, below, overcome some of these disadvantages.

(b) Evidence using "substrata" formed in vitro. Decidual stromal cells form "substrata" when cultured in vitro (10). These substrata consist of the cell surfaces and their associated ECM. We have reported that macrophage-mediated tumor lysis, as one measure of macrophage function, is inhibited on decidual substrata. In contrast, tumor lysis on substrata formed by fibroblasts is not inhibited (10). Contact between macrophage and the substratum was required for the regulatory ef-

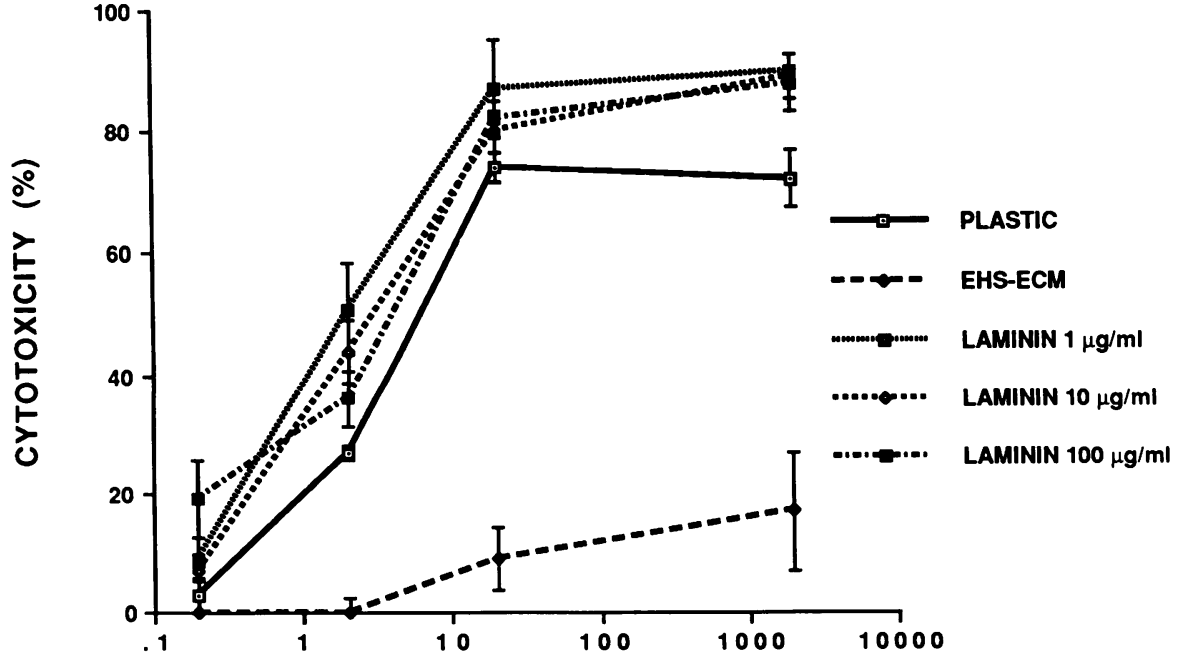

ENDOTOXIN DOSE $(\mathrm{ng} / \mathrm{ml})$
Figure 6. Laminin does not inhibit macrophage-mediated tumor lysis. RAW 264.7 macrophage were cultured upon wells coated with the indicated amounts of laminin, the gel formed by EHS ECM, or directly upon tissue culture plastic ( $P L A S$ $T I C)$. All macrophages were stimulated by $100 \mathrm{U} / \mathrm{ml} \mathrm{rIFNg}$ and the indicated doses of endotoxin. ${ }^{11}$ In-labeled P815 were added and the tumor cytotoxicity determined. Each value is the mean and standard error of three determinations. 


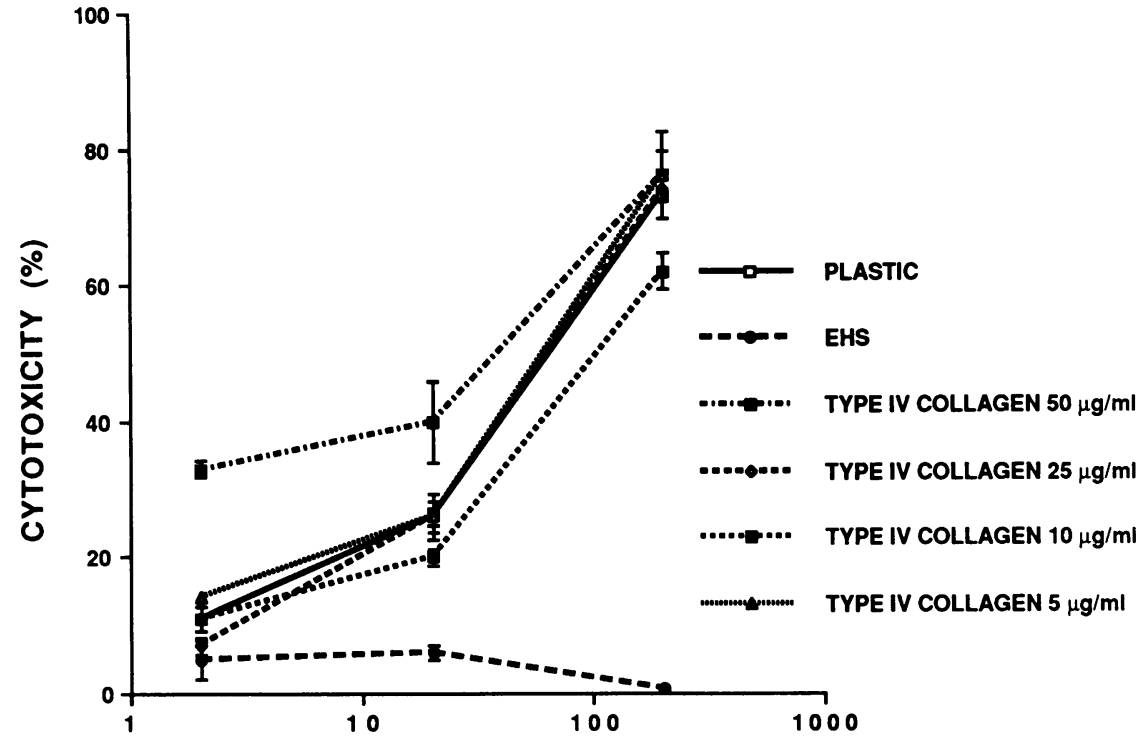

ENDOTOXIN DOSE $(\mathrm{ng} / \mathrm{ml})$
Figure 7. Type IV collagen does not inhibit macrophage-mediated tumor lysis. RAW 264.7 macrophages were cultured as in Fig. 6 except that the wells were coated with the indicated amounts of Type IV collagen. Each value is the mean and standard error of three determinations. fects. This data is consistent with the hypothesis that decidual ECM is inhibitory for macrophage activation, whereas interstitial ECM formed by fibroblasts is permissive and extends the studies done in vivo.

(c) Evidence using ECM in vitro. When the component molecules of decidual and EHS ECMs are brought to physiologic ionic strength, $\mathrm{pH}$, and temperature, a gel forms. This is thought to represent reconstitution of the ECM in vitro. Fig. 1 shows that both decidual and EHS ECMs inhibit macrophagemediated tumor lysis when compared to a gel formed by Type I collagen, which is a model for interstitial ECM. Fig. 2 indicates that contact between ECM and macrophage is necessary for the regulatory effects. These results extend the substratum data of the previous paragraph by showing that it is the ECM rather than the cell surfaces which are permissive or inhibitory for macrophage functions. Note that tissue culture plastic was also used as a model for a permissive surface (Figs. 2-4, 6-9) because it is presently the most widely used surface where macrophage functions do occur in vitro, and is thus a standard, allowing comparisons with the published literature.

The two inhibitory ECMs, decidual and EHS, have a number of common properties. Both have, as major constituents,

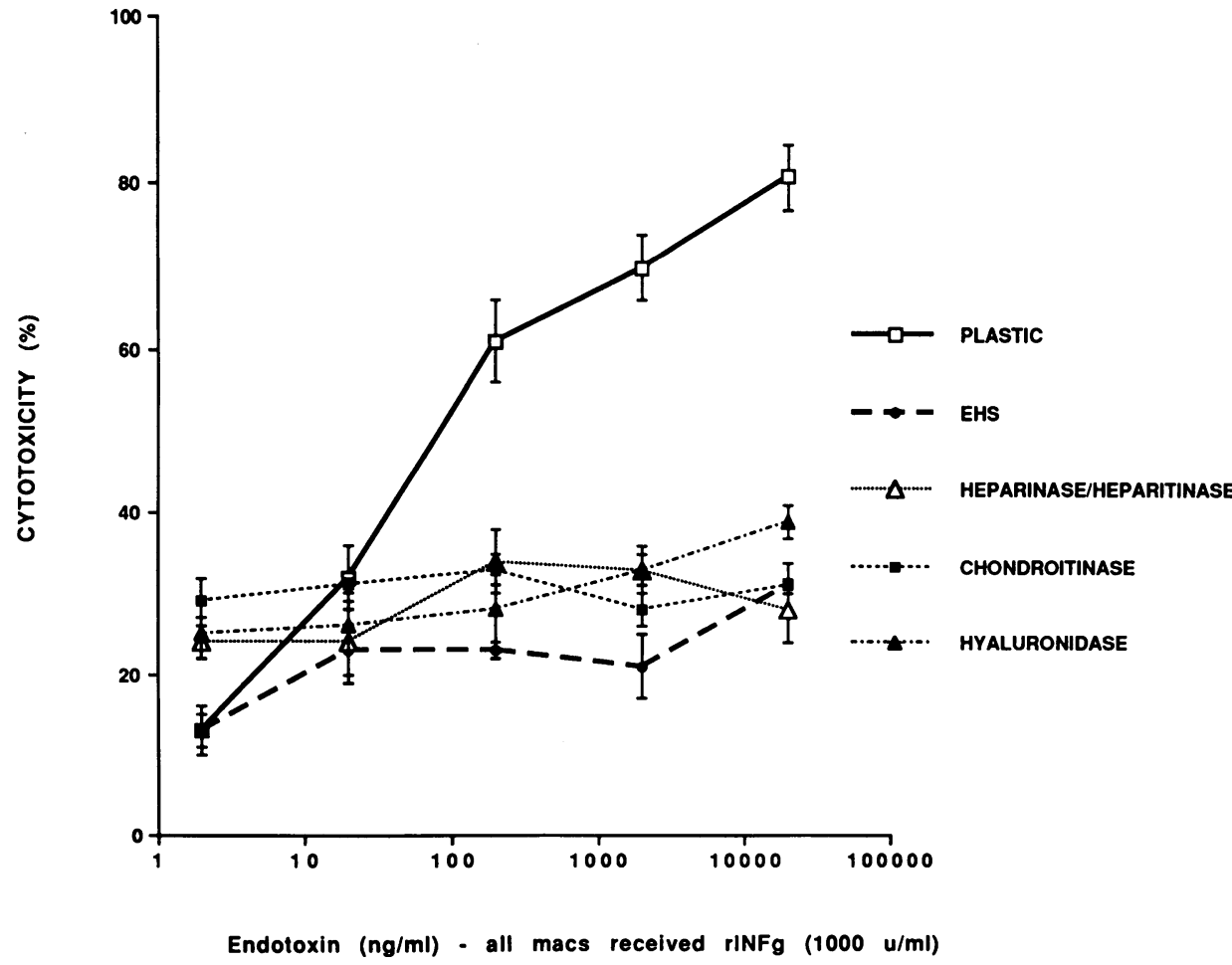

Figure 8. Treating EHS ECM gels with heparinase/heparitinase $(\mathrm{H} / \mathrm{H})$, chrondroitinase $\mathrm{ABC}$, or hyaluronidase does not prevent inhibition of macrophage-mediated tumor lysis. Gels formed by EHS ECM were exposed to mixture of heparinase and heparitinase (open triangles), chrondroitinase ABC (solid squares), hyaluronidase (solid triangles) or media (solid circle). RAW 264.7 macrophages were cultured upon these gels or tissue culture plastic (open squares), stimulated with rIFNg (100 $\mathrm{U} / \mathrm{ml}$ and the indicated doses of endotoxin, and allowed to lyse ${ }^{111}$ Inlabeled P815 tumor cells. Each value is the mean and standard error triplicate determinations. 


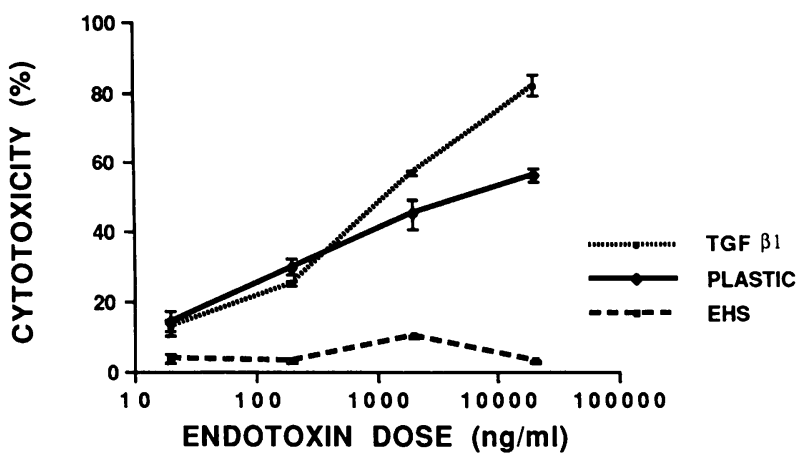

Figure 9. TGF-beta 1 does not inhibit macrophage-mediated tumor lysis. RAW 264.7 macrophages residing on tissue culture plastic were stimulated with $100 \mathrm{U} / \mathrm{ml}$ rIFNg and the indicated doses of endotoxin (LPS), and allowed to lyse ${ }^{111}$ In-labeled P815 tumor cells. 100 $\mathrm{ng} / \mathrm{ml}$ TGF-beta 1 (TGF-B1, dotted line, solid circle) or media (PLASTIC, solid line, solid circle) were added to these macrophages. Another group of macrophages resided upon gels formed by EHS ECM. These macrophages were also stimulated by rIFNg and endotoxin, but did not receive TGF beta 1 ( $E H S$, dashed line, solid squares).

basement membrane type macromolecules (11-13). Both inhibit the effector phase of macrophage-mediated tumor lysis (see Fig. 3 and reference 10). Antilisterial responses in vivo are inhibited in decidual and EHS tissues (Fig. 5 and references 8 , 9): Future studies will determine if the active inhibitors within these two inhibitory ECMs are actually the same molecules.

The precise biochemical identification of the inhibitor(s) in decidual and EHS ECMs is beyond the scope of this report. However, Figs. 6 and 7 indicate that laminin and type IV collagen, two major known components of these ECMs, are not inhibitory by themselves. A large basement-membrane heparan sulfate proteoglycan is another major component of decidual and EHS ECMs (11-13). However, Fig. 8 shows that treating EHS ECM with heparinase and heparitinase did not abrogate inhibition of macrophage-mediated tumor lysis. This result must be interpreted cautiously. We cannot, at this time, exclude the possibilities that the enzymes failed to remove critical heparan sulfate glycosaminoglycans, or that the heparan sulfate core protein has inhibitory activity even after removal of the glycosaminoglycans. However, in a similar model system, intact glycosaminoglycan-core protein linkages are important in the inhibitory effect of dermatan sulfate proteoglycan on fibroblast adhesion to fibronectin-coated surfaces (36).

Recent evidence indicates that some growth factors, such as FGFb and IL3 (for review see reference 37), bind to ECM by virtue of ionic interactions with the highly negative heparan sulfate proteoglycans. In those models, the ECM was a vehicle containing the regulator molecule. We believe an inhibitor bound by charge interactions is unlikely in decidual and EHS ECM because such inhibitors should be removed by the washes of insoluble ECM with $3.4 \mathrm{M} \mathrm{NaCl}, \mathrm{pH} 7.4$, during the purification procedure.

PGE2, immune complexes, and endotoxin are not responsible for the inhibitory effect of EHS ECM. PGE2, which has been reported to inhibit macrophage-mediated tumor lysis (38), is soluble in aqueous solutions and should have been removed during the preparation of the EHS ECM. The tumor is washed with saline, dialyzed against saline and media at $4^{\circ} \mathrm{C}$, and washed extensively with media after the gels are formed (see Methods). Furthermore, media conditioned by the EHS ECM gels should contain any soluble inhibitors, but was not inhibitory (see Fig. 2). Using the limmulus amebocyte assay, we find $<0.02 \mathrm{ng} / \mathrm{ml}$ endotoxin in our EHS ECM preparations because of meticulous technique (see Methods). In any event, endotoxin would augment rather than inhibit macrophage-mediated tumor lysis. See Figs. 4, 6-9, where increasing amounts of endotoxin increased tumor lysis. Although insolubilized antigen-antibody can inhibit macrophage-mediated tumor lysis (39), such complexes should have been disrupted during the preparation of EHS ECM which included exposure to $3.4 \mathrm{M}$ $\mathrm{NaCl}$ and $2 \mathrm{M}$ urea.

Proteases may contribute to macrophage-mediated tumor lysis. Such proteases are thought to be secreted into spaces between macrophage and tumor cell and where the plasma membranes of these cells contact one another (40). Only soluble small molecular weight inhibitors such as bovine pancreatic trypsin inhibitor (mol wt 6,300) can enter these spaces and prevent tumor lysis. Large serum antiproteases such as alpha2-macroglobulin cannot enter these spaces and are not inhibitory $(34,40)$. The EHS ECM inhibitor is not soluble (see Fig. 2 ), would not enter these spaces, and therefore is not an antiprotease.

Insofar as decidual and EHS ECMs contain, as major constituents, type IV collagen, laminin, and heparan sulfate proteoglycans, they are members of the broad class of basement-membrane ECMs. Despite these similarities, the various basement-membrane ECMs do differ from one another functionally, structurally, and biochemically in the presence or absence of minor components $(41,42)$. At the present time, we cannot exclude the possibility that other basement-membrane ECMs, in addition to decidua and EHS, may also inhibit macrophage functions. This issue is under study in our laboratory. However, even if all basement membranes did tend to inhibit macrophage functions, decidual and EHS ECMs are a much more substantial barriers for macrophages on the basis of greater thickness alone. The decidua and the EHS tumor are many cells thick, and these cells are embedded in basementmembrane ECM. Most other basement membranes, on the other hand, are delicate, thin, sheetlike structures lining epithelia or endothelia, and are only several microns thick.

Although the biochemical nature of the solid phase inhibitor of macrophage function remains to be defined, its presence in decidual and EHS ECM may be important from several standpoints. Solid phase regulators within the ECM have previously been shown to regulate functions of a number of cell types (for example, see references 43,44 ), but inhibition of macrophage functions by this pathway has not, to our knowledge, been previously reported. Understanding this inhibitory pathway may be important in elucidating the pathogenesis of perinatal infections and the maternal-fetal immunologic relationship in the murine decidua basalis.

Furthermore, our observations may be important in understanding the complex interactions between malignant tumors and their hosts. Although macrophages are clearly capable of killing many types of tumors, some tumors survive, in part, because they inhibit macrophage functions. Previously described "deactivators" of macrophage function secreted by some tumors have been soluble molecules (for examples, see references 25,45$)$. To our knowledge, this paper is the first report that solid phase molecules in a tumor ECM inhibit macrophage functions and thus contributes to tumor survival. Our 
data suggest that the EHS tumor and decidua share common mechanisms in inhibiting macrophage functions. Indeed, tumors and fetal cells in the decidua, the latter by virtue of their paternal alloantigens vis-à-vis the maternal host, also share the property of surviving in a potentially hostile host immunologic environment.

\section{Acknowledgments}

This work was supported by grants from the National Institute of Health (RO-1-HD-24797 and RO1-DK-43634), the Hood Foundation, the Hearst Foundation, and the March of Dimes. Dr. McKay was supported by a training grant awarded to the Renal Division, Brigham and Women's Hospital, from the National Institutes of Health (2T32DK-07527-03). Dr. Redline was the recipient of a NIH Clinical Investigator Award (KO-8-HD-00864). Dr. Vazquez was supported by the Kidney and Pancreatic Transplant Grant from the State of Texas, and subsequently by an individual research fellowship from the National Institutes of Health (F32-AI08290-01). Dr. Lu was a Faculty Fellow of the Hartford Foundation and subsequently recipient of a NIH Research Career Development Award (K04-HD-00862).

\section{References}

1. Redline, R. W., and C. Y. Lu. 1989. Localization of fetal major histocompatibility complex (MHC) antigens and maternal leukocytes in the murine placenta: implications for understanding the regulation of maternal anti-fetal immune responses. Lab. Invest. 61:27-36.

2. Lu, C. Y., R. W. Redline, D. B. McKay, L. B. Dustin, and C. M. Shea. 1989. Regulation of macrophage functions in the murine placenta and decidua: implications for tolerance of the fetal allograft. Transplant. Rev. 3:195-214.

3. Hunziker, R. D., and T. G. Wegmann. 1986. Placental immunoregulation. CRC Crit. Rev. Immunol. 6:245-285.

4. Clark, D. A., A. Chaput, R. M. Slapsys, J. Brierley, S. Daya, and R. Allardyce. 1987. Suppressor cells in the uterus. In Immunoregulation and fetal survival. T. J. Gill III and T. G. Wegmann, editors. Oxford University Press, New York. 63.

5. Strom, T. B., J. W. Kupiec-Weglinski, and N. L. Tilney. 1985. On the mechanisms of rejection of vascularized organ allografts: review and an attempt at synthesis. Prog. Transplant. 2:126-146.

6. Hahn, H., and S. H. E. Kaufmann. 1981. The role of cell-mediated immunity in bacterial infections. Rev. Inf. Dis. 3:1221-1250.

7. Redline, R. W., and C. Y. Lu. 1987. Role of local immunosuppression in murine fetoplacental listeriosis. J. Clin. Invest. 79:1234-1241.

8. Redline, R. W., and C. Y. Lu. 1988. Specific defects in the anti-listerial immune response in discrete regions of the murine uterus and placenta account for susceptibility to infection. J. Immunol. 140:3947-3955.

9. Redline, R. W., C. M. Shea, V. E. Papaioannou, and C. Y. Lu. 1988 Defective anti-listerial responses in deciduoma of pseudopregnant mice. Am. J. Pathol. 133:485.

10. Redline, R. W., D. B. McKay, V. E. Papaioannou, C. M. Shea, and C. Y. Lu. 1990. Macrophage functions are regulated by the substratum of decidualized endometrial stromal cells. J. Clin. Invest. 85:1951-1958.

11. Damjanov, I. 1985. Vesalius and Hunter were right: decidua is a membrane. Lab. Invest. 53:597-598.

12. Wewer, U. M., A. Damjanov, J. Weiss, L. A. Liotta, and I. Damjanov. 1986. Mouse endometrial stromal cells produce basement-membrane components. Differentiation. 32:49.

13. Kleinman, H. K., M. L. McGarvey, J. R. Hassell, and G. R. Martin. 1983. Formation of a supramolecular complex is involved in the reconstitution of basement membrane components. Biochemistry. 22:4969-4974.

14. Cheers, C., and I. F. C. McKenzie. 1978. Resistance and susceptibility of mice to bacterial infection: genetics of listeriosis. Infect. Immun. 19:755-762.

15. Skamene, E., P. A. L. Kongshavn, and D. H. Sachs. 1979. Resistance to Listeria monocytogenes in mice: genetic control by genes that are not linked to the H-2 complex. J. Inf. Dis. 139:228-234.

16. Kleinman, H. K., M. L. McGarvey, J. R. Hassell, V. L. Star, F. B. Cannon, G. W. Laurie, and G. R. Martin. 1986. Basement membrane complexes with biological activity. Biochemistry. 25:312-318.

17. Weinberg, J. 1981. Endotoxin contamination and in vitro monocytemacrophage function: methods of detecting, detoxifying, and eliminating endotoxin. In Methods for Studying Mononuclear Phagocytes. D. O. Adams, P. J. Edelson, and H. Koren, editors. Academic Press, Inc., New York. 139-154.

18. Kennedy, T. G. 1986. Intrauterine infusion of prostaglandins and decidualization in rats with uteri differentially sensitized for the decidual cell reaction. Biol. Reprod. 34:327-335.
19. Raschke, W. C., S. Baird, P. Ralph, and I. Nokoinz. 1978. Functional macrophage cell lines transformed by Abelson leukemia virus. Cell. 15:261.

20. Gorecka-Tisera, A. M., K. W. Snowdowne, and A. B. Borle. 1986. Implications of a rise in cytosolic free calcium in the activation of RAW 264.7 macrophages for tumor cell killing. Cell. Immunol. 100:411.

21. Austyn, J. M., and S. Gordon. 1981. F4/80 a monoclonal antibody directed specifically against the mouse macrophage. Eur. J. Immunol. 11:805.

22. Decker, T., M. L. Lohmann-Matthes, and G. E. Gifford. 1987. Cell-associated tumor necrosis factor (TNF) as a killing mechanism of activated cytotoxic macrophages. J. Immunol. 138:957-962.

23. North, R. J. 1981. An introduction to macrophage activation. Lymphokines. 3:1-24.

24. Adams, D. O., and T. A. Hamilton. 1984. The cell biology of macrophage activation. Annu. Rev. Immunol. 2:283-318.

25. Harrell, R. A., G. J. Cianciolo, T. D. Copelannd, S. Oroszlan, and R. Snyderman. 1988. Suppression of the respiratory burst of human monocytes by a synthetic peptide homologous to envelope proteins of human and animal retroviruses. J. Immunol. 136:3517-3520.

26. Tsunawaki, S., and C. F. Nathan. 1986. Macrophage deactivation: altered kinetic properties of superoxide-producing enzyme after exposure to tumor-cell conditioned medium. J. Exp. Med. 164:1319-1331.

27. Inaba, K., M. Kitaura, T. Kato, Y. Watanabe, and S. Muramatsu. 1986. Contrasting effect of alpha/beta and gamma-interferons on expression of macrophage Ia antigens. J. Exp. Med. 163:1030-1035.

28. Lambert, L. E., and D. M. Paulnock. 1989. Differential induction of activation markers in macrophage cell lines by interferon gamma. Cell. Immunol. 120:401-418.

29. Russell, S. W., G. Y. Gillespie, and J. L. Pace. 1980. Comparison of responses to activating agents by mouse peritoneal macrophages and cells of the macrophage line RAW 264. J. Reticuloendothel. Soc. 27:607-619.

30. Clark, D. A., M. Falbo, R. B. Rowley, D. Banwatt, and J. StedronskaClark. 1988. Active suppression of host-vs-graft reaction in pregnant mice. IX. Soluble suppressor activity obtained from allopregnant mouse decidua that blocks the cytolytic effector response to IL-2 is related to transforming growth factor-beta. J. Immunol. 141:3833-3840.

31. Altman, D. J., S. L. Schneider, D. A. Thompson, H. L. Cheng, and T. B. Tomasi. 1990. A transforming growth factor beta2 (TGF b2)-like immunosuppressive factor in amniotic fluid and localization of TGF-b2 mRNA in the pregnant uterus. J. Exp. Med. 172:1391-1402.

32. Roberts, A. B., and M. B. Sporn. 1988. Transforming growth factor beta. Adv. Cancer Res. 51:107-146.

33. Celada, A., and R. D. Schreiber. 1986. Role of protein kinase C and intracellular calcium mobilization in the induction of macrophage tumoricidal activity by interferon-gamma. J. Immunol. 137:2372-2379.

34. Cybulsky, M. I., and M. A. Gimbrone, Jr. 1991. Endothelial expression of a mononuclear leukocyte adhesion molecule during atherogenesis. Science (Wash. DC). 251:788-791.

35. Haskill, S., C. Johnson, D. Eierman, S. Becker, and K. Warren. 1988. Adherence induces selective mRNA expression of monocyte mediators and proto-oncogenes. J. Immunol. 140:1690-1694.

36. Lewandowska, K., H. U. Choi, L. C. Rosenberg, L. Zardi, and L. A. Culp. 1987. Fibronectin-mediated adhesion of fibroblasts: inhibition by dermatan sulfate proteoglycan and evidence for a cryptic glycosaminoglycan binding domain. J. Cell Biol. 105:1443-1454.

37. Finn, C. A. 1971. The biology of decidual cells. Adv. Reprod. Physiol. $5: 1-26$.

38. Schultz', R. M., N. A. Pavlidis, W. A. Stylos, and M. A. Chirigos. 1978. Regulation of macrophage tumoricidal function: a role for prostaglandins of the E series. Science (Wash. DC). 202:320-321.

39. Esparza, I., R. Green, and R. D. Schreiber. 1983. Inhibition of macrophage tumoricidal activity by immune complexes and altered erythrocytes. $J$. Immunol. 131:2117-2121.

40. Adams, D. O., K. J. Kao, R. Farb, and S. V. Pizzo. 1980. Effector mechanisms of cytolytically activated macrophages. II. Secretion of a cytolytic factor by activated macrophages and its relationship to secreted neutral proteases. $\mathrm{J}$. Im munol. 124:293-300.

41. Klein, D. J., D. M. Brown, T. R. Oegema, P. E. Brenchley, J. C. Anderson, M. A. J. Dickinson, E. A. Horigan, and J. R. Hassell. 1988. Glomerular basement membrane proteoglycans are derived from a large precursor. J. Cell Biol. 106:963-970.

42. Yurchenco, P. D., and J. C. Schittny. 1990. Molecular architecture of basement membranes. FASEB (Fed. Am. Soc. Exp. Biol.) J. 4:1577-1590.

43. McClay, D. R., and C. A. Ettensohn. 1987. Cell adhesion in morphogenesis. Annu. Rev. Cell Biol. 3:319-345.

44. Tan, S. S., K. L. Crossin, S. Hoffman, and G. M. Edelman. 1987. Asymmetric expression in somites of cytotaxin and its proteoglycan ligand is correlated with neural crest cell distribution. Proc. Natl. Acad. Sci. USA. 84:7977.

45. Tsunawaki, S., M. Spron, A. Ding, and C. F. Nathan. 1988. Deactivation of macrophages by transforming growth factor-beta. Nature (Lond.). 334:260262. 